\title{
Effects of shock, ego-threat, and neutral conditions on affective experience during paired-associates learning'
}

MARTIN KATAHN AND DONALD F. PAGANO

VANDERBILT UNIVERSITY

\begin{abstract}
Subjective ratings of affective experience made by Ss engaged in a paired-associates learning experiment were not differentially affected by shock, ego-threat, or neutral instruction conditions. Ss with high scores on the Taylor Manifest Anxiety Scale rated themselves as experiencing significantly more negative affect than did low anxious Ss. After receiving instructions and immediately after learning (but not during learning itself) Ss receiving ego-threat and shock instructions tended to feel more negative affect than did Ss in the neutral condition, but not significantly so. No interaction of anxiety by instruction conditions was obtained. Problem
\end{abstract}

This study was concerned with the effects of various stress inducing conditions on the subjective experience of college students while performing on a pairedassociates learning task. Lazarus et al (1952) suggest that stress can be considered either ego-oriented or task-oriented. They define ego-oriented stress as a threat to the maintenance of one's integrity, e.g., the use of instructions which relate performance to intelligence or which emphasize that failure will reflect other forms of personal inadequacy. Task-oriented stress is compared to a barrier in the environment which is to be overcome, e.g., the threat of shock.

Little is known at the present time regarding the effects of these various forms of stress induction upon subjective experience. Using scales based upon Wundt's three dimensions of feeling (Wundt, 1907), Mordkoff (1963) found high correlations between subjective experience and physiological indices of stress. This suggests that such subjective reports might prove to be useful in-

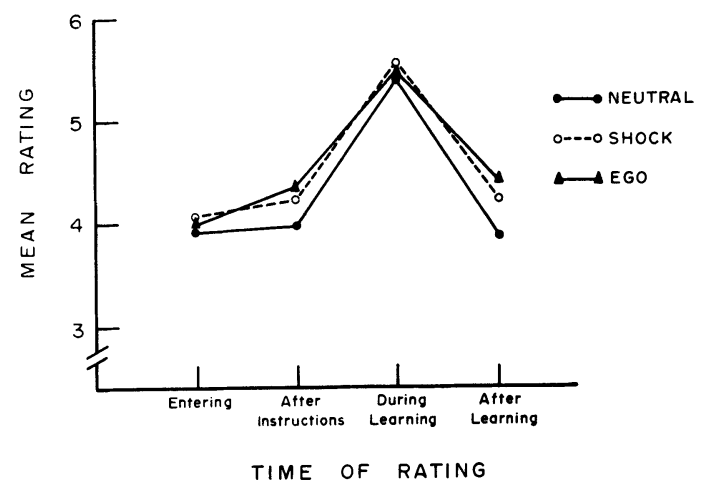

Fig. 1. Mean ratings on combined affect scales for each instruction group. dicators of reaction to different experimental conditions. The first purpose of this experiment was to investigate the relationship of subjective reactions to two forms of stress induction as well as to the standard instructions commonly used in paired-associates learning.

The second purpose was to determine whether any relationship exists between subjective reactions, i.e., reported negative affect, and manifest anxiety. There is accumulated evidence that high anxious (HA) Ss are inferior to low anxious (LA) Ss in certain complex learning tasks (Sarason, 1960), especially when some form of ego-threatening instructions is employed. Are these performance differences also paralleled by higher negative affective ratings during the experiment?

Method and Proeedure

Fifty-one female Ss from psychology classes at Vanderbilt University participated, with $17 \mathrm{Ss}$ serving in each experimental condition. For anxiety comparisons, 15 Ss with Taylor Manifest Anxiety Scale (MAS) scores below 12, and 15 Ss with scores above 23 comprised LA and HA subgroups, respectively, and the remaining 21 Ss made up the middle anxiety group (MA). The $\mathrm{N}$ within cells for anxiety by conditions comparisons was proportional.

Upon entering the individual testing situation, each $S$ rated herself on eight-point scales labelled to reflect Wundt's three affect dimensions: relaxed-tense, quiet-excited, and pleasant-unpleasant. Group N (neutral) then received standard instructions for a paired-associates (P-A) learning task; Group E (ego-threat) was shown manufactured data and graphs which related P-A learning to success in college; Group S (shock) received instructions embodying the threat of shock for wrong responses. Ss in this group did, in fact, receive a moderate shock for the first wrong response on the second and fourth trial of a difficult P-A list in which no associative strength existed between any of the words according to all commonly used norms. Only six trials were given on the list, since the concern of the study was upon experience and not learning.

Ss rerated themselves after receiving instructions and then performed on the learning task. Immediately following the last learning trial, Ss rated how they felt while performing on the P-A task and then did a final "now" rating on the three scales of feeling.

\section{Results and Diseussion}

As in Mordkoff's (1963) study, ratings on the three affect scales changed in a similar manner at all rating points. Therefore, following his procedure, ratings were combined for purposes of analysis. In Fig. 1, mean rating magnitudes of the combined affect scales for each instruction group are plotted as a function of time of rating. The higher the rating, the more negative the affect. An analysis of variance yielded non-significant $F^{\prime} \mathrm{S}$ for the main effect of experimental conditions and the interaction of conditions with time of rating ( $F^{\prime} S=1$ or less). For time of rating itself, $F=14.79, p<.001$.

It may be seen that all Ss rated themselves initially at about the same level. After receiving instructions and upon completion of the experiment the $\mathrm{S}$ and $\mathrm{E}$ 
groups rated themselves as experiencing slightly more negative affect than the $\mathrm{N}$ group, which showed little change from its initial rating. When Ss rated themselves on their feelings during the task, the three instruction groups showed a remarkable similarity. One explanation for this lack of difference may be that the experimental conditions did not provide enough of a threatening situation for $\mathrm{Ss}$ in the $\mathrm{S}$ and $\mathrm{E}$ groups. The rating scales themselves seem to have provided enough ceiling to allow for differences, since mean ratings fell considerably below the ceiling value of "8." The S and E groups, however, did indicate more negative feelings after receiving instructions than did the $\mathrm{N}$ group. Thus, it seems likı. that factors unrelated to instructions began to operate during learning itself, motivating all groups to indicate about the same average degree of negative affect. This raises some question regarding the extent to which various threat conditions differentially affect the subjective experience of Ss required to participate in a difficult task. So-called "stress conditions" may add little to the degree of negative affect experienced by most Ss operating under the standard or "neutral" conditions in many psychological experiments. Further research seems necessary to clarify this issue.

The mean affect ratings of the high, middle and low anxiety groups (instructions and scales combined) are illustrated in Fig. 2. The main effects of anxiety were significant $(\mathrm{F}=3.29, \mathrm{df}=2 / 42, \mathrm{p}<.05)$. Deleting the MA $\mathrm{Ss}$, the difference between the HA and LA groups alone was even more significant $(F=6.61, \mathrm{df}=1 / 24, \mathrm{p}<.025)$. Except for ratings during learning itself (when MA Ss rated themselves slightly higher than HASs), the higher the anxiety score, the greater the expression of negative affect. HA Ss rated themselves generally as experiencing more negative affect in all treatment conditions, and no interaction of anxiety by treatments was obtained. There was a slight tendency for HA Ss to report relatively more negative affect than LA Ss in the S condition, rather than in the $\mathrm{E}$ condition. Similar differences in overall affective ratings between $\mathrm{HA}$ and LA Ss have now been obtained in an entirely different verbal learning experiment in which a buzzer was used to indicate wrong responses (Katahn. \& Lyda, 1965).

While high scores on the MAS may simply reflect a more favorable attitude toward the expression of negative feelings (a test response bias), the close correlation between ratings and physiological indices reported by Mordkoff and the significantly greater increase in heart-rate on the part of test-anxious Ss in a difficult learning situation reported by Harleston et al (1965) seem to suggest that HA Ss actually do experience more negative affect and greater physiological arousal than LA Ss while performing in difficult learning situations.

As for learning itself, the significant difference in the ratings of affective experience by the three anxiety groups was not paralleled by a significant difference in actual performance, although HA Ss tended to be slightly inferior to MA and LA Ss. This tendency is consistent

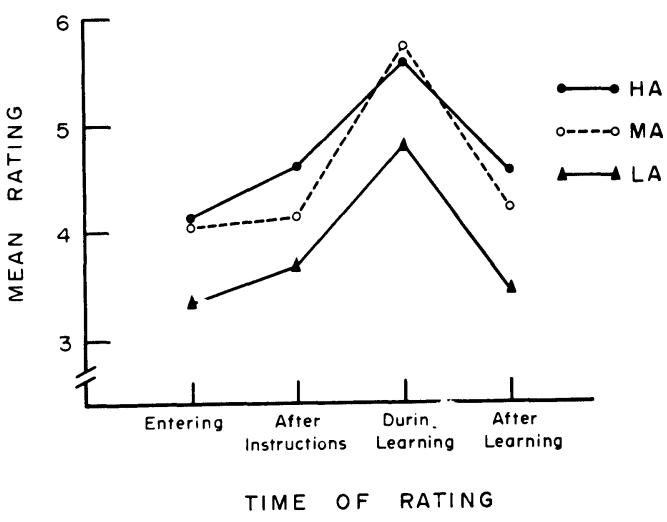

Fig. 2. Mean ratings on combined affect scales for high, medium, and low anxious subjects.

with previous findings using a difficult P-A list (e.g., Spence et al, 1956). Although instruction conditions did not significantly affect learning, $\mathrm{Ss}$ in the $\mathrm{E}$ group gave the greatest number of correct responses and Ss in the $S$ group gave the fewest. Since both these groups indicated somewhat more negative affect before and after learning, it is obvious that no direct relationship exists between affective experience and performance, at least within the range of experience and conditions reported in this study.

Finally, since certain studies (Sarason, 1960) have reported that ego-threat is most detrimental to performance for HA Ss, it seems important to note that just the opposite tendency was obtained in the present experiment. While HA Ss as a group were inferior to the other Ss, HA Ss in the E condition learned better than $\mathrm{HA}$ Ss in the $\mathrm{S}$ and $\mathrm{N}$ conditions, which is in line with the general facilitating effect of egothreat instructions for all Ss in the sample. This may be due to the fact that especially stringent admission requirements for females at Vanderbilt probably tend to exclude students who perform poorly in a threatening, competitive academic environment.

\section{References}

Harleston, B. W., Smith, M. G., \& Arey, D. Test anxiety level, heart rate, and anagram problem solving. J. Pers. soc. Psychol., $1965,1,551-557$.

Katahn, M., \& Lyda, L. L. Anxiety and the learning of responses varying in initial rank in the response hierarchy. Unpublished manuscript, Department of Psychology, Vanderbilt University, 1965.

Lazarus, R. S., Deese, J., \& Osler, S. J. The effects of psychological stress upon performance. Psychol. Bull., 1952, 49, 293-417.

Mordkoff, A. M. The relationship between psychological and physiological response to stress. A paper read before Western Psychological Association, 1963.

Sarason, G. Empirical findings and theoretical problems in the use of anxiety scales. Psychol. Bull., 1960, 57, 403-415.

Spence, K. W., Farber, I. E., \& McFann, H. H. The relation of anxiety (drive) level to performance in competitional and noncompetitional paired-associates learning. J. exp. Psychol., 1956, 52, 306-310.

Wundt, W. Outlines of psychology. Translated by Judd, 3rd rev. English ed. from 7th rev. German ed., 1907.

Note

1. This research was supported by Public Health Service Grants No. MH-08784 and MH-11149, National Institutes of Health. 